\title{
Some issues of calculation of complex rod and plate systems on an elastic base
}

\author{
Oksana Kozunova ${ }^{1,2^{*}}$ \\ ${ }^{1}$ Belarusian State University of Transport, 246653 Gomel, Belarus \\ ${ }^{2}$ Belarusian National Technical University, 220013 Minsk, Belarus
}

\begin{abstract}
This paper summarizes some issues of a new universal method for static calculation of complex rod and plate systems on an arbitrary elastic base under the influence of an external load, when modeling the base with various models, ranging from the classical Winkler model to the combined Winkler-Kogan model.

The relevance and timeliness of the proposed work lies in the fact that the issues of calculating complex structures on an elastic base have not yet been fully investigated and there is no general approach to their calculation. The author knows the works of M. I. Gorbunov-Posadov, I. A. Simvulidi, B. N. Zhemochkin, G. Ya. Popov, S. N. Klepikov, S. D. Semenyuk, S. V. Bosakov, in which various approaches have been used to conduct research on the calculation of simple core structures, isolated foundation structures, including mesh slabs, as well as spatial monolithic foundations, as a system of cross belts on an elastic base.

The proposed method is based on a mixed method of structural mechanics using the functions of the influence of an elastic medium in the relations of B. N. Zhemochkin. To simplify these relations, when determining the coefficients of canonical equations and free terms of the mixed method in the calculation of isolated and connected plates, the relations for deflections of plates with a normal pinched in the centre of the plate are used. For pivotally connected beams, it is convenient to introduce pinching with unknown displacements at the beginning or end of each beam, for frame rods-in their nodal connections. The numerical implementation of the general approach is performed using the Mathematica computer program.
\end{abstract}

\section{Introduction}

From the history of the development of static calculations of rod and plate systems on an elastic base, it can be seen that the methods of calculating the above structures were improved and refined as a result of scientific and technological progress [1-6]. The variety of practical problems leads to an ambiguous modeling of the elastic base. A particular difficulty is the choice of a computational model of an elastic base due to its inhomogeneity, layering and physical nonlinearity for plastic connected soils. An overview

\footnotetext{
*Corresponding author: kozunova@gmail.com
} 
of elastic base models for the calculation of foundation beams, beam and foundation plates is given in [7,8]. Models of elastic foundations with further practical applications for the static calculation of cross bands of shallow foundations are successfully systematized and classified in the monograph by S. D. Semenyuk [9].

If at the initial stage they were content with the hypothesis of a linear distribution of reactive stresses $[1,2,5]$, then during the rapid construction of railway tracks, as well as pontoon structures, the Fuss-Winkler-Zimmerman model was developed. However, the latter did not satisfy the real work of connected soils. This is how the elastic half-space model appeared (with the spatial formulation of the problem), and the well-known Boussinesq solution for this model [2], and with the flat formulation - the elastic half-plane model with the Fleman solution [2,8], respectively. In the future, various modifications and combinations of these models appeared. The combination of the elastic half-space model and the Winkler model is very successfully suitable for calculating structures on inhomogeneous bases with a layered structure. Such bases are found in the construction of foundations on a sand cushion and are modeled by combined models [10, 11].

From a mechanical point of view, the calculation of rod and plate systems on an elastic base is a solution to the contact problem of touching bodies [12]. These problems are reduced to solving integral equations, the solution of which depends on the kernel of the integral equation and the shape of the contacting bodies [13]. For simple forms, the main difficulty is to determine the law of distribution of contact stresses $[6,12,13]$. In engineering practice, it is impractical to solve every contact problem through integral equations due to time-consuming mathematical calculations. Therefore, in practice, the Zhemochkin method is successfully used [14], which reduces the contact problem to the problem of structural mechanics [15].

The issues of static calculation of complex rod and plate systems on an elastic base have not yet been fully investigated, there is no general universal approach to this calculation. The author is familiar with the works of M. I. Gorbunov-Posadov [2], I. A. Simvulidi [16], G. Ya. Popov [17], S. N. Klepikov [4], S. D. Semenyuk [9], S. V. Bosakov [6] in which studies on the static calculation of building structural systems and their elements on an elastic base were carried out by various approaches.

Below, the author suggests a general approach of structural mechanics for the static calculation of such complex systems, such as:

1) articulated rectangular plates on a combined Winkler-Kogan base, the model of which is proposed and tested in the works of the author $[11,18]$;

2) articulated foundation beams based on Winkler, taking into account the physical nonlinearity of the beam material [19];

3) articulated flat frames on an elastic Winkler base and on an elastic half-space [20];

4) mesh foundation plates on a linearly deformable base, the calculation of which was numerically tested for an elastic half-space [21] and for an elastic isotropic layer [22].

\section{The essence of the general approach}

The essence of the proposed general approach is as follows: a complex rod or plate system is divided into separate elements in the form of a beam, a simple frame and a rectangular plate. Each element on an elastic base is calculated by the Zhemochkin method for an external load and unknown forces in the connections of the elements with each other. A joint system of resolving equations is obtained, where the forces in the Zhemochkin connections at the contact of each element with an elastic base, the linear and angular displacements of the introduced pinches on each element, as well as the forces in the connections between the elements are unknown. Further, the usual methods of structural 
mechanics [15] determine the displacements and internal forces in the elements of the system.

The numerical implementation of the proposed approach is carried out using examples of complex rod and plate structures symmetrically loaded with a vertical load on various models of an elastic base using the Mathematica computer program.

The idea of B. N. Zhemochkin's method and its development: from simple to complex. The paper summarizes the method of static calculation of complex composite rod and plate systems on an arbitrary elastic base, having any shape in plan and loaded with an arbitrary external load. It is known that for a linearly deformable medium, the principle of independence of the action of forces and the linear dependence between loads and displacements are valid. The calculation of structures is carried out in the traditional formulation without taking into account tangential forces on the contact surface of the plate, and the validity of the hypotheses of bending of thin plates. The method used in the calculations is based on a combination of the method of B. N. Zhemochkin and a mixed method of structural mechanics, proposed earlier in $[6,9,14]$ for calculating isolated structures of simple and complex shape in plan.

For a better understanding of the general approach to calculating complex rod and plate systems on an arbitrary elastic base, the following is the method of B. N. Zhemochkin $[6,14]$ for calculating a simple beam lying on an elastic base under the influence of an external load (Fig.1). As a result, it is necessary to determine the values of reactive pressures in the contact zone of the beam with elastic warping, the distribution of internal forces (bending moments and transverse forces in its sections), precipitation and rotation angles.

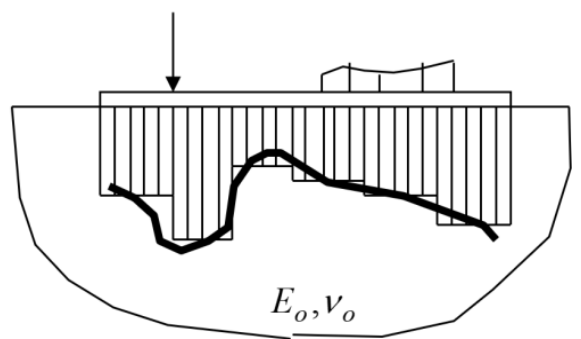

Fig. 1. Replacement of the jet plot the pressure of the step line

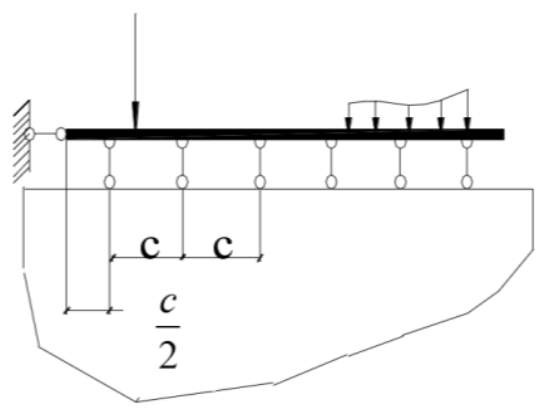

Fig. 2. Arrangement of connections B. N. Zhemochkin in the contact zone

According to the method of B. N. Zhemochkin, the beam is divided into equal sections along its length, and absolutely rigid connections are placed in the middle of each section, through which the beam contacts the elastic base (Fig. 2). The horizontal rod in Fig.2 is set to fulfill the condition of geometric immutability and when only a vertical load is applied, the force in it is zero. It is assumed that the force in each connection causes a uniform plot of reactive pressures within each section. Thus, from the calculation of a beam on an elastic 
base (Fig.1), it is necessary to proceed to the calculation of a statically indeterminate beam connected to an elastic base by rigid bonds at individual points. To solve this problem, a mixed method is chosen, taking as an unknown the forces in the introduced connections, the linear and angular displacement of the pinching introduced on the beam (Fig. 3).

Canonical equations of the mixed method are compiled, expressing the conditions for the compatibility of the deformations of the beam and the elastic base.

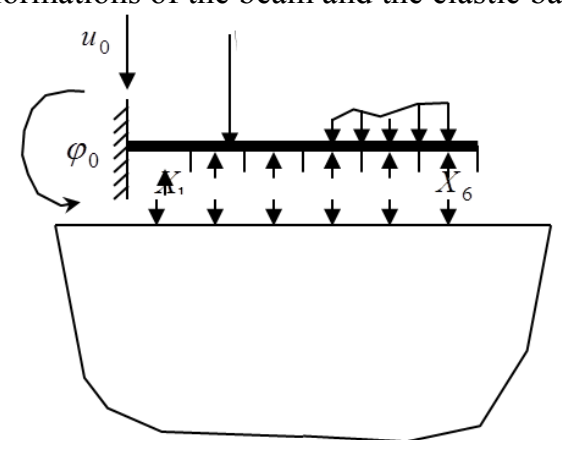

Fig. 3. The main system method of B. N. Zhemochkin

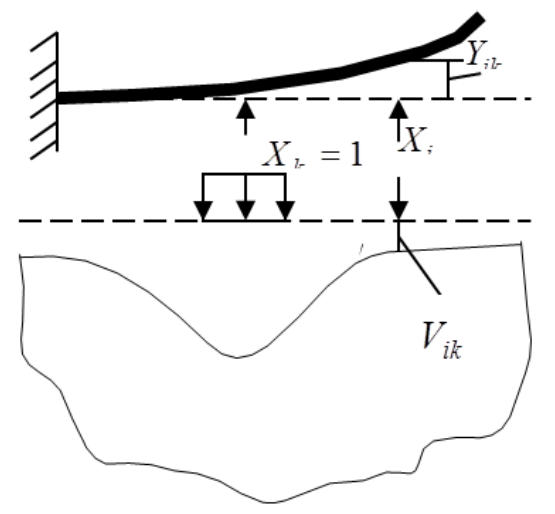

Fig. 4. To determine the coefficients equations of the method of B. N. Zhemochkin

The equilibrium equations are added to these equations. The system of linear algebraic equations of the method of B. N. Zhemochkin is obtained

$$
\left\{\begin{array}{l}
\delta_{11} X_{1}+\delta_{12} X_{2}+\cdots+\delta_{16} X_{6}+\varphi_{o} \frac{c}{2}+u_{o}+\Delta_{1 p}=0 \\
\delta_{21} X_{1}+\delta_{22} X_{2}+\cdots+\delta_{26} X_{6}+\varphi_{o} \frac{3 c}{2}+u_{o}+\Delta_{2 p}=0 \\
\cdots \\
\delta_{61} X_{1}+\delta_{62} X_{2}+\cdots+\delta_{66} X_{6}+\varphi_{o} \frac{11 c}{2}+u_{o}+\Delta_{6 p}=0 \\
-\frac{c}{2} X_{1}-\frac{3 c}{2} X_{2}-\cdots-\frac{11 c}{2} X_{6}+M_{p}=0 \\
-X_{1}-X_{2}-\cdots-X_{6}+R_{p}=0
\end{array}\right.
$$

where $M_{p}, R_{p}$ - the moment of external forces relative to the pinching and the resultant of external forces;

$\Delta_{i p}-$ vertical displacement of the point $i$ of the main system (pinched beam) from the external load; 
$u_{0}, \varphi_{0}-$ linear and angular displacement of the introduced pinching at the edge of the beam (Fig. 3).

The coefficients for unknown forces in the connections of B. N. Zhemochkin in the system (1) depend on the deflections of the beam in the main system of the mixed method (Fig. 3) and the sediment of the elastic base, and are determined by the formula

$$
\delta_{i k}=Y_{i k}+V_{i k},
$$

where $Y_{i k}$-deflection of the beam at the point of application $X_{i}$ in the main system from the action $X_{k}=1$ (Fig. 4);

$V_{i k}$ - moving the application point $X_{i}$ at the boundary of the elastic base from the action of a length evenly distributed over the site $c$ force $X_{k}=1$ (Fig. 4).

The replacement of a concentrated force with a distributed load is caused by the fact that the displacements of the elastic base at the point of application of the concentrated force are unlimited. In the case of a spatial problem for a beam of finite width, B.N. Zhemochkin assumes that the reactive pressure is the same across the width of the beam and a rigid connection is placed in the center of a rectangular section of the beam with the size $b^{*} c$, where $b$ - beam width.

In expression (2), the displacement $V_{i k}$ is determined by the type of elastic base. For example, for the elastic half-space model [6]

$$
V_{i k}=\frac{1-V_{0}^{2}}{\pi E_{0} c} F_{i k}^{0}
$$

where $F_{i k}^{0}$ - a dimensionless function for determining the vertical displacement of the point $i$ of the elastic half-space boundary from the action of a uniformly distributed over a rectangular section $k$ of the base boundary by the dimensions $b^{*} c$ of a unit force. Its expression is given in [17]. There you can also find numeric values for $F_{i k}^{0}$ depending on the size of the rectangle $b^{*} c$ and the border points where the displacement is determined.

If the Winkler model is adopted for the elastic base, then in (2) it is necessary to take [6]

$$
\begin{aligned}
& V_{i k}=0, \quad i \neq k, \\
& V_{i k}=\frac{1}{K b c}, \quad i=k,
\end{aligned}
$$

where $K$ - bed coefficient of elastic foundation.

After solving system (1), the bending moments and transverse forces in the beam sections are determined by the values of the reactive forces $X_{k}$ found. At the same time, it is advisable to consistently go from the free edge of the main system to the pinching (Fig. 3). Since the deflections of the beam on the elastic base are identically equal to the precipitation of the elastic base, it can be written to determine the deflections of the point $k$

$$
Y_{k}=\sum_{i=1}^{n} X_{i} V_{i k}
$$

where $n-$ the number of plots of B.N. Zhemochkin on the structure.

At this point, the solution to the problem can be considered complete.

If we consider an isolated road, foundation or mesh slab on an elastic foundation, then the resolving system of equations will be 


$$
\left(\begin{array}{l}
\sum_{i=1}^{n}\left(\sum_{k=1}^{n} \delta_{i k} X_{k}+\varphi_{0 x} y_{i}+\varphi_{0 y} x_{i}+u_{0}+\Delta_{i p}=0\right) \\
-\sum_{k=1}^{n} X_{k} y_{k}+M_{p x}=0 \\
-\sum_{k=1}^{n} X_{k} x_{k}+M_{p y}=0 \\
-\sum_{k=1}^{n} X_{k}+Q=0
\end{array}\right.
$$

where $u_{0}, \varphi_{0 x}, \varphi_{0 y}{ }^{-}$linear and angular displacements of the introduced pinching on the plate;

$Q, M_{p x}, M_{p y},-$ resultant of external forces acting on the plate and its moments relative to the coordinate axes.

In relations (5), the coefficients at unknowns $\delta_{i k}$ and free terms $\Delta_{i p}$ will depend on the deflections of the slab with the clamped normal and the shape of the section B.N. Zhemochkin.

The method of B.N. Zhemochkin was further developed in the works of S.D. Semenyuk [9], S.V. Bosakov. [6,11, 19, 21-26,28], Dmitrieva (Yurkova) K.V. [23], Skachek P.D. [2426], Bartolomei L.A.,.Cernant A.A. [27] and in the works of the author [11, 18-22].

About the combined (three-layer) model of the elastic base. The elastic base model in the form of a combined model was proposed by the author in collaboration with Bosakov S.V., Zinevich S.I. in [11] to calculate an isolated road plate of highways and airfields on an inhomogeneous (layered) base. The model of such a base is represented as a Winkler base (a layer of crushed stone) located on a two-layer Kogan base (sand + natural ground) (Fig.5), and can be defined as a combined Winkler-Kogan model.

Using the results of the work of B.I. Kogan [10] and S.V. Bosakov [6] on determining the displacements of a two-layer base, as well as the approach of B.N. Zhemochkin [14] on calculating a rigid plate on a two-layer base, an expression was obtained in the author's coauthorship [11] to determine the displacements of a point on the surface of a combined (three-layer) base from the action of a load evenly distributed over a rectangular section of the surface of a three-layer base.

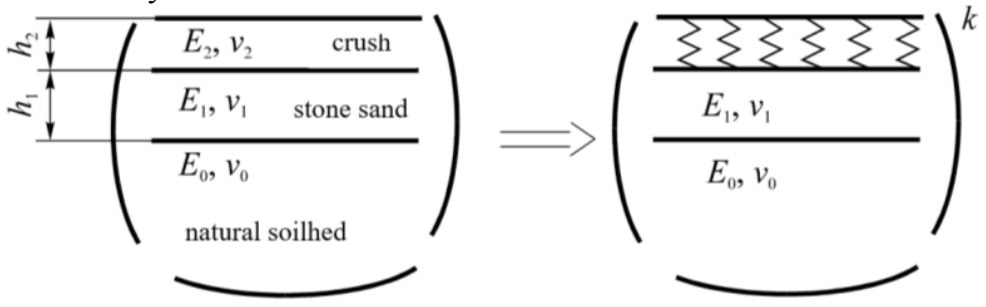

Fig. 5. Combined model of elastic base

The combined base model is close to the real engineering and geological conditions of the construction site and can be used to calculate articulated rectangular slabs on heterogeneous (layered) bases.

\section{General statement of the problems of contact interaction}


For complex plate (plate) systems and mesh plates, each plate or tape is divided into identical rectangular sections in size $\Delta x \times \Delta y$ and a vertical connection is introduced in the centre of each section, through which the plate contacts the elastic base.

Similarly to plate systems for complex rod systems in the form of articulated beams or frames, each beam (frame) is divided into sections of equal length and a vertical connection is introduced in the center of each section, through which the beam (frame) contacts the elastic base. According to the method of B.N. Zhemochkin, it is believed that the force in connection causes a uniform pressure plot within the site.

When setting tasks, hypotheses and assumptions are introduced for contacting bodies and contact zones:

- it is assumed that only normal stresses act on the contact of the structure with the base [2];

- for plates and beams, the hypotheses of the bending theory are valid [15], and for frames and mesh plates, torsion must be taken into account;

- connecting hinges - cylindrical;

- the distribution of contact stresses over the width of the structure is uniform.

The resulting statically indeterminate system is solved by a mixed method of structural mechanics [15], taking for unknown forces in the introduced connections, linear and angular movements of the pinched normals in the center of each plate, beam (frame) or at the edges of beams (frames) and transverse forces in the connecting hinges (see the algorithm for the corresponding calculation below in problem 1 ).

\subsection{Problem 1}

A flat frame is considered as a system of variously connected rod elements on an elastic base under the action of an external load, which form a closed contour. The design model of a flat frame on an elastic base is shown in Fig.6, and its division (dismemberment) into separate rod elements, pivotally connected into a system, in Fig. 7.

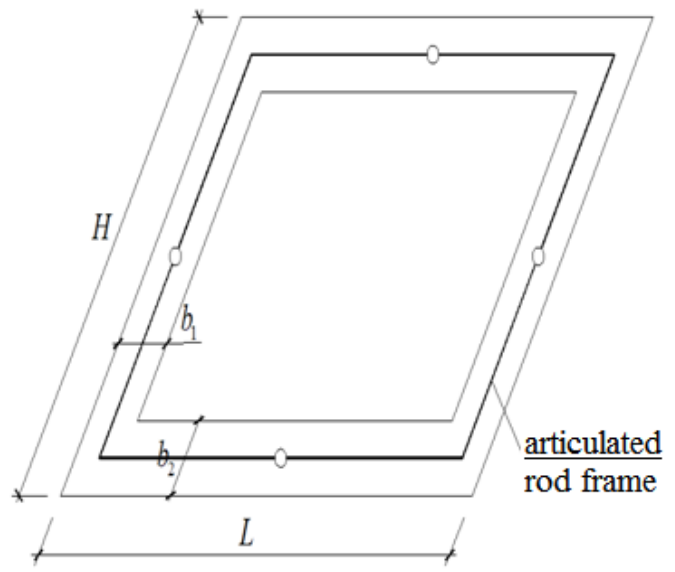

Fig. 6. Calculation model of a flat frame 


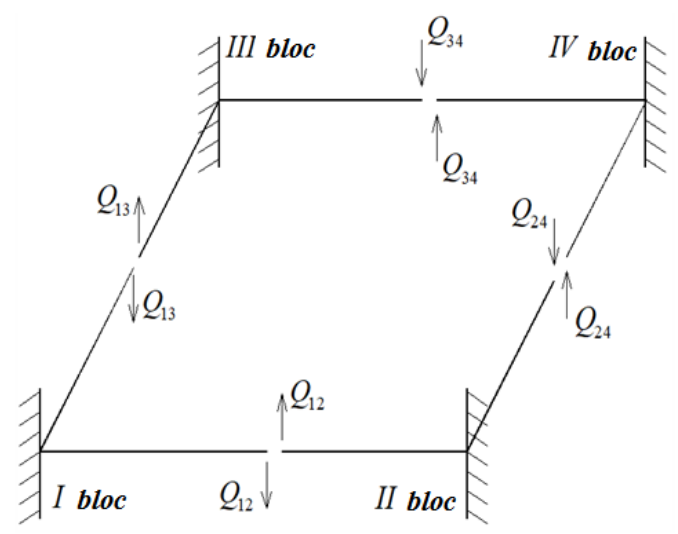

Fig. 7. Splitting a flat frame into L-shaped rod elements connected by hinges

I would like to note that in this example, the flat frame is divided into blocks according to the number of pivotally connected rod elements (see Fig.7), the places of their connection are modeled by cylindrical hinges (see Fig.6), which, when forming the main system, will be replaced by a pair of unknown transverse forces $Q_{i k}$, fictitious seals of the mixed method are introduced into rigid nodes.

In the static calculation of flat frames on an elastic base, the following are determined: contact stresses under the rod elements of the frame, forces in their cross-sections and precipitation of the base under the entire flat frame.

Calculation algorithm. Each rod of the flat frame is divided into identical rectangular sections and in the center of each section (the sections lie on the axes of the frame) a vertical connection is introduced through which the contact of the flat frame with the elastic base is carried out (Fig.8). It is believed that the force in the connection causes a uniform pressure plot within the section.

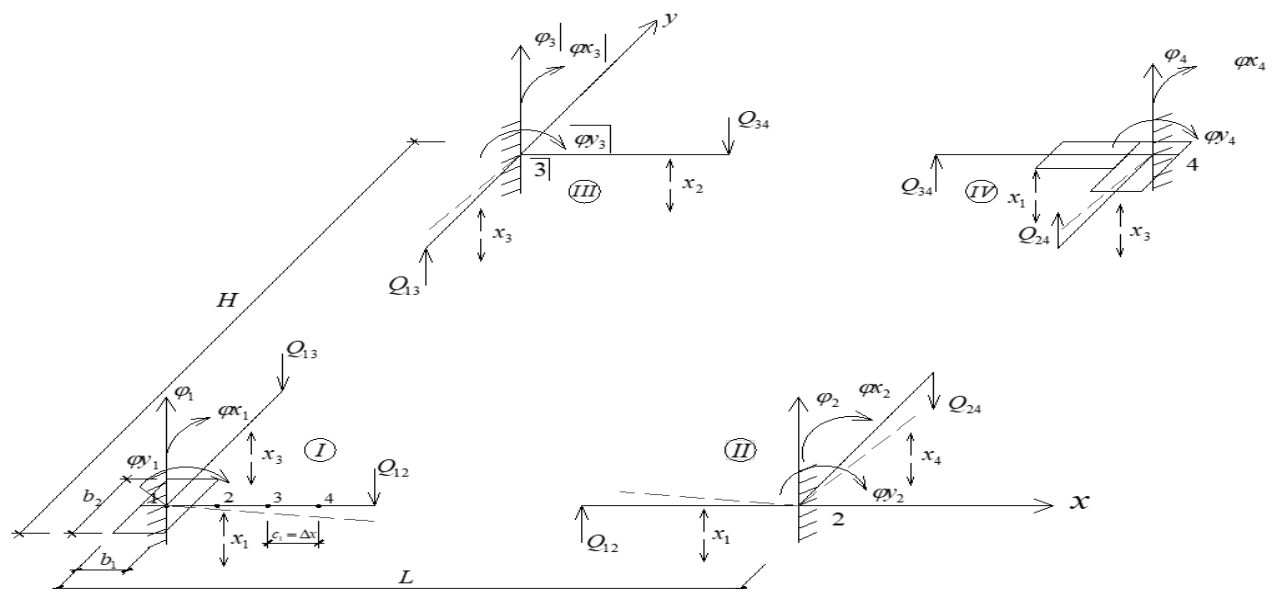

Fig. 8. The basic system of the mixed method

The resulting repeatedly statically indeterminate system is solved by a mixed method of structural mechanics [15], as before, taking as unknown: the forces $X_{k}$ in the introduced connections of B.N. Zhemochkin at the contact of the frame and base elements, linear and angular displacements $u_{k}, \varphi_{k}$ pinches introduced in the corners of the frame or in the center of the rods, depending on the locations of the intermediate hinges, and transverse forces $Q_{i k}$, 
in these hinges. Note that there are no torques in the places of cuts under the action of a symmetrical load.

Some features of the division into Zhemochkin sections arise in the corners of the frame (Fig.9) due to the modeling of a flat frame by a system of rod elements.
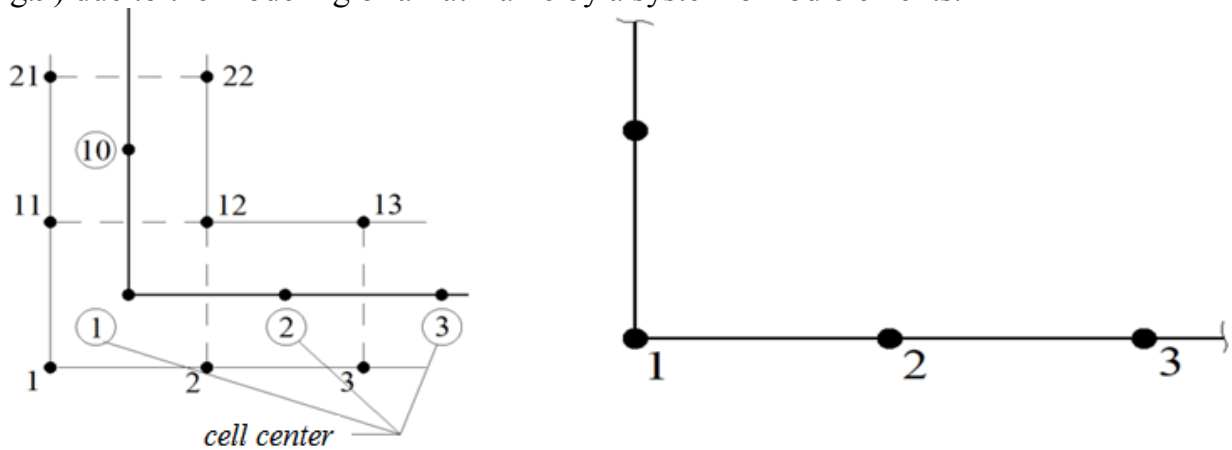

Fig. 9. Features of the division into sections of Zhemochkin in the corners of the frame

The canonical equations of the mixed method for the block of the main system with the number «i» are written in the following form

$$
\left\{\begin{array}{l}
\sum_{k=1}^{2} \delta_{Q, i+1} Q_{i+1}^{k}+\delta_{1,1} X_{1}+\ldots+\delta_{1, m} X_{m}+u_{i}+\varphi x_{i} y_{1}+\varphi y_{i} x_{1}+\sum_{k=1}^{2} \delta_{Q, i+2} Q_{i+2}^{k}+\Delta_{1, p}=0 \\
\ldots \\
\sum_{k=1}^{2} \delta_{Q, i+1} Q_{i+1}^{k}+\delta_{m, 1} X_{1}+\ldots+\delta_{m, m} X_{m}+u_{i}+\varphi x_{i} y_{m}+\varphi y_{i} x_{m}+\sum_{k=1}^{2} \delta_{Q, i+2} Q_{i+2}^{k}+\Delta_{m, p}=0 \\
\sum_{k=1}^{m} X_{k} y_{k}-\frac{H}{2} Q_{i, i+1}-M_{x}=0 \\
\sum_{k=1}^{m} X_{k} x_{k}-\frac{L}{2} Q_{i, i+2}-M_{y}=0 \\
\sum_{k=1}^{m} X_{k}-Q_{i, i+1}-Q_{i, i+2}-R=0 \\
\sum_{k=1}^{2} \delta_{Q, i+1} Q_{i+1}^{k}+u_{i-1}-\frac{h}{2} \varphi x_{i-1}-\frac{L}{2} \varphi y_{i-1}+u_{i}-\frac{h}{2} \varphi x_{i}+\frac{L}{2} \varphi y_{i}=0 \\
\sum_{k=1}^{2} \delta_{Q, i+1} Q_{i+1}^{k}+u_{i-1}+\frac{h}{2} \varphi x_{i-1}+\frac{L}{2} \varphi y_{i-1}+u_{i}+\frac{h}{2} \varphi x_{i}-\frac{L}{2} \varphi y_{i}+\sum_{k=1}^{2} \delta_{Q, i+2} Q_{i+2}^{k}=0
\end{array}\right.
$$

where $m-$ is the number of Zhemochkin sections on an L-shaped rod element connected by hinges (block "i" of the main system);

$u_{\mathrm{i}}, \varphi x_{i}, \varphi y_{i}-$ unknown linear and angular displacements of the introduced pinching on an L-shaped rod element connected by hinges (block "I" of the main system);

$R, M_{x}, M_{y}-$ resultant of external forces and resultant moments relative to coordinate axes on an L-shaped rod element connected by hinges (block "I" of the main system);

$Q_{i, i+1}, Q_{i, i+2}$ - transverse forces in split hinges on an L-shaped rod element connected by hinges (block "I" of the main system);

$X_{\mathrm{k}}$ - effort in connection with Zhemochkin 's number $k$.

The blocks along the main diagonal are formed according to the system of canonical equations of the mixed method for calculating one element with the number $i$, the side blocks are zero in the case of the Winkler base. In the case of an elastic half-space or an elastic layer, they characterize the mutual influence of the frame blocks and are determined using formulas from Bosakov S.V. monograph [6]

a) for the Winkler foundation 


$$
\begin{aligned}
& \delta_{i, k}=\frac{1}{K c b}+\frac{b^{2}}{16 \pi D} w_{i, k}, \quad i=k \\
& \delta_{i, k}=\frac{b^{2}}{16 \pi D} w_{i, k}, \quad i \neq k,
\end{aligned}
$$
$Y$;

where $c$ - the length of Zhemochkin's section, $c=\Delta x$ and $c=\Delta y$ along the acting axes $X$,

$b$ - the width of Zhemochkin's section, $b=b_{1}$ and $b=b_{2}$ along the acting axes $Y$ and $X$ respectively;

b) for an elastic half-space

$$
\delta_{i, k}=\frac{1-v_{0}^{2}}{\pi E_{0} \Delta x} F_{i, k}+\frac{b^{2}}{16 \pi D} w_{i, k} .
$$

where the dimensionless function of the influence of the elastic medium $F_{i, k}$ is determined by the formula [6]

$$
\begin{aligned}
F_{i, k}= & \frac{y_{i}-d}{\Delta y} \ln \frac{x_{i}-b+\sqrt{\left(x_{i}-b\right)^{2}+\left(y_{i}-d\right)^{2}}}{x_{i}-a+\sqrt{\left(x_{i}-a\right)^{2}+\left(y_{i}-d\right)^{2}}}+\frac{y_{i}-c}{\Delta y} \ln \frac{x_{i}-a+\sqrt{\left(x_{i}-a\right)^{2}+\left(y_{i}-c\right)^{2}}}{x_{i}-b+\sqrt{\left(x_{i}-b\right)^{2}+\left(y_{i}-c\right)^{2}}}+ \\
& +\frac{x_{i}-b}{\Delta y} \ln \frac{y_{i}-d+\sqrt{\left(x_{i}-b\right)^{2}+\left(y_{i}-d\right)^{2}}}{y_{i}-c+\sqrt{\left(x_{i}-b\right)^{2}+\left(y_{i}-c\right)^{2}}}+\frac{x_{i}-a}{\Delta y} \ln \frac{y_{i}-c+\sqrt{\left(x_{i}-a\right)^{2}+\left(y_{i}-c\right)^{2}}}{y_{i}-d+\sqrt{\left(x_{i}-a\right)^{2}+\left(y_{i}-d\right)^{2}}}
\end{aligned}
$$

where $i-$ is the number of the Zhemochkin plot, where the movements are determined; $a, b, c, d$ - coordinates of the Zhemochkin section with the number " $k$ ", where a uniformly distributed load with a single resultant is applied.

The first $(m+3)$ equations of system (1) are similar to the standard equations of the mixed method in the relations of the Zhemochkin method [6,14], which additionally include 2 transverse forces in the split hinges. The last two equations express the conditions for the absence of vertical mutual displacements at the locations of intermediate hinges on the border of two adjacent blocks of a flat frame.

The free terms of the system (1) are determined from the external load and depend on its type, namely: the distributed load determines all the components of the column vector in the matrix of the system of linear algebraic equations to be nonzero, the concentrated force is applied at the nodes.

If we denote the number of blocks of a flat frame by $N_{p}$, then the total number of unknown forces in the Zhemochkin connections, linear and angular displacements of the introduced pinches on an L-shaped rod element connected by hinges, and transverse forces in the hinges will be expressed by the formula

$$
N_{c}=N_{p}(m+3)+2 N_{p}-4 .
$$

Calculation results. Calculations were carried out for a flat reinforced concrete frame of individual manufacture with dimensions $L=8 \mathrm{~m} \times H=8 \mathrm{~m}$, band width $-b=b_{1}=b_{2}=1 \mathrm{~m}$ made of heavy concrete $C^{20 / 25}$ on an elastic foundation, modeled by the Winkler base with the bed coefficient $K=200 \mathrm{MPa} / \mathrm{m}$ and an elastic half - space with the following characteristics of the base $E_{0}=20 \mathrm{MPa}, v_{0}=0,3$. External load: concentrated force $P=10 \mathrm{kN}$, attached in the corners of the frame, and evenly distributed load $q=10 \mathrm{kN} / \mathrm{m}$ on all frame rods.

Figure 10 shows the numbering of the Zhemochkin sections in a flat frame, which is also symmetrical in a symmetrical frame. You should pay attention to the corner element, 
which is numbered twice: number 1 - for the horizontal direction, number 5 - for the vertical. The concentrated force was entered into the calculation as $P / 2$ for each.

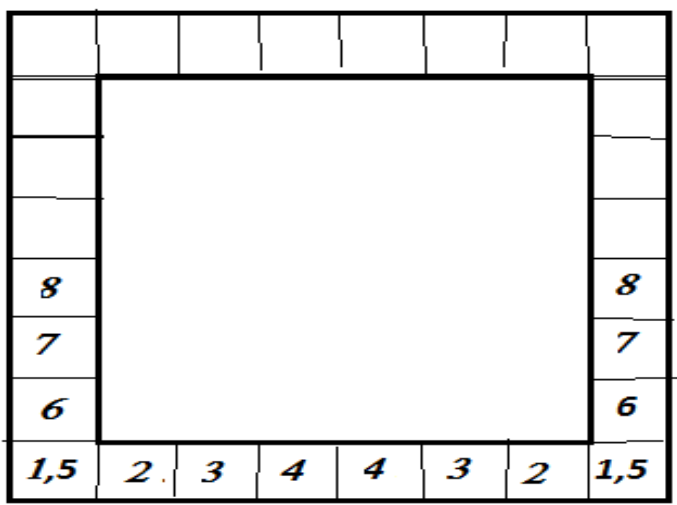

Fig. 10. Numbering of Zhemochkin sections in a flat frame.

In Figure 11, plots of the sediment of the elastic base under the lower band of the frame from the concentrated force in the corners of the frame are constructed when modeling the elastic base a) by the Winkler base, b) by the elastic half-space.

a)

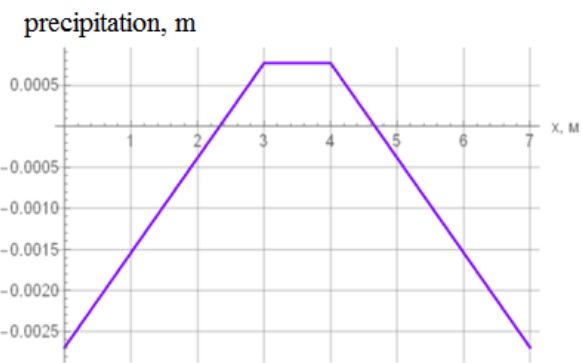

b)

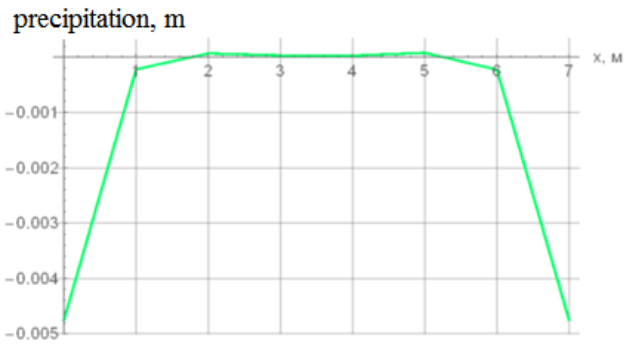

Fig. 11. Precipitation of the elastic base $(\mathrm{m})$ under the lower band of the frame with numbers $1,5 \ldots .44 \ldots .1 .5$ of the concentrated force in the corners of the frame: a) - on the basis of Winkler; b) on an elastic half-space.

In Figure 12, plots of the sediment of the elastic base under the lower band of the frame from the distributed load are constructed when modeling the elastic base a) the Winkler base, b) the elastic half-space.
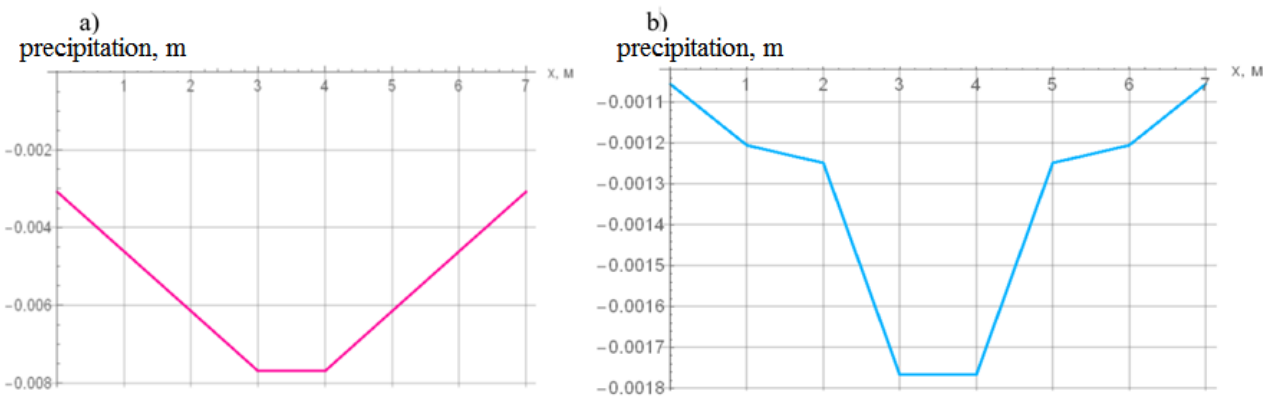

Fig. 12. Precipitation of the elastic base $(m)$ under the lower band of the frame with numbers 1,5...4_4....1.5 of the distributed load: a) - on the basis of Winkler; b) - on an elastic half-space. 


\section{Conclusions}

The paper considers some issues of static calculation of articulated road slabs on a combined elastic base, articulated foundation beams on a Winkler base, taking into account the physical nonlinearity of the beam material, a hinged flat frame on a Winkler base and on an elastic half-space, as well as foundation mesh slabs on an elastic half-space and a homogeneous isotropic layer. The method for all the above calculations is universal, based on a mixed method of structural mechanics and the method of B. N. Zhemochkin, and can be generalized to any number of plates, beams and frames of finite rigidity, based on various models of an elastic base. The above approach is easily generalized to the calculation of complex rod and plate systems on an elastic base, taking into account the physical nonlinearity of the construction material.

The difference from the standard Zhemochkin method for simple beams is to take into account additional unknown transverse forces and torques at the joints of the rod elements of each block and to compile compatibility equations for linear and angular displacements. It should be noted the following: when taking into account the torques, the plot of contact stresses along the width of the frame rod, for example, has an asymmetric appearance. When using the Zhemochkin method, the average value of the contact voltage across the width of the rod is obtained. This unevenness is not reflected on the values of bending moments in the cross sections of the frame rods. However, the author believes that more accurate results can be achieved when calculating a flat frame on an elastic base, as a mesh system [13, 14], where the rod is replaced by a plate with its division of at least two Zhemochkin sections along the width of the plate. But this requires a separate study and is not considered in this paper.

According to the results of the calculation, many complex construction objects were designed and erected by the method of B. N. Zhemochkin. However, the potential of this method is not completely exhausted, and therefore one can find examples [23,27,28] of its use for analytical calculation of problems of the theory of elasticity and verification of the results of numerical methods.

\section{References}

1. M. I. Gorbunov-Posadov, Beams and plates on an elastic base (1949)

2. M. I. Gorbunov-Posadov, T. A. Malikova, V. I. Solomin, Calculation of structures on an elastic base, issue 3 (1984)

3. B. G Korenev, Questions of calculation of beams and plates on an elastic base (1954)

4. S. N. Klepikov, Calculation of structures on an elastic base (1967)

5. L. A. Galin, Development of the theory of contact problems in the USSR (1976)

6. S. V. Bosakov, Static calculations of plates on an elastic base, (2002)

7. A. N. Tarasevich, Bending of self-stressed plates on an elastic base, dis. Candidate of Technical Sciences : 05.23 .17 (2001)

8. O. V. Kozunova, Static analysis of the system "beam plate-nonlinear elastic inhomogeneous base" by the variational-difference method, dis. Candidate of Technical Sciences: 05.23.17 (2017)

9. S. D. Semenyuk, Reinforced concrete spatial foundations of residential and civil buildings on an unevenly deformable base (2003)

10. B. I. Kogan, Stresses and deformations of multilayer coatings, issue 14, 33-46 (1953) 
11. S. V. Bosakov, S. I. Zinevich, O. V. Kosanova, A model for the elastic Foundation and its use for the calculation of rectangular plates on elastic Foundation, Scientifictechnical journal: Structural mechanics and calculation of structures, № 4, 2 -5 (2018)

12. A.V. Alexandrov, V. D. Potapov, Fundamentals of the theory of elasticity and plasticity: for educational building. special universities, 2nd ed., (2002)

13. A. B. Vasilyeva [et al.], Differential and integral equations, calculus of variations in examples and problems (2003)

14. B. N. Zhemochkin, A. P. Sinitsyn, Practical methods of calculations of foundation beams and plates on an elastic base, 2nd ed., (1962)

15. R. A. Rzhanitsyn, Construction mechanics (1991)

16. I. A. Simvulidi, Calculation of engineering structures on an elastic base (1987)

17. G. Ya. Popov, On the calculation of an unlimited hinged-split beam plate lying on an elastic half-space, Construction and Architecture, 3, 25-33 (1959)

18. O. V. Kozunova, Using the model of a three-layer base in the calculations of articulated road slabs, Bulletin BrSTU, №1, 44-52 (2020)

19. S. V. Bosakov, O. V. Kosanova, Development of the theory of calculation pivotally connected beams on elastic Foundation based on their physical nonlinearity, Problems of modern concrete and reinforced concrete, Vol. 11, 11-24 (2019)

20. O. V. Kozunova, Some problems of analysis of plane frames on elastic Foundation on the spatial load, Structural mechanics and calculation of structures, №4, 13-22 (2021)

21. S. V. Bosakov, O. V. Kozunova Development of the theory of the calculation mesh of plates on elastic foundation, Structural mechanics and calculation of structures, №3, 20-25 (2020)

22. S. V. Bosakov, O. V. Kozunova, Calculation of foundation mesh plates on an elastic layer, Problems of modern concrete and reinforced concrete, Vol.12, 11-27, (2020)

23. K. V. Dmitrieva (Yurkova), Calculation of a rigid wall in an elastic weighty half-plane №6, tome 15, 493-503 (2016) [DOI: 10.21122/2227-1031-2016-15-6-493-503]

24. S. V. Bosakov, P. D. Skachek, Solution of spatial contact problems for the hinge of the unit bearing single-span beams, Structural mechanics and calculation of structures, №4, 10-19 (2019)

25. S. V. Bosakov, P. D. Skachek, Determination of contact stresses at the hinge bearing beams on the edge of the wall, Structural mechanics and calculation of structures, №6, 33-40 (2020)

26. S. V. Bosakov, P. D. Skachek, The solution of the spatial contact problem of the hinge nodes of the beam support on elastic quarter-space and one-eighth of space, Problems of modern concrete and reinforced concrete, Vol.12, 28-44 (2020)

27. L. A. Bartholomey, N. Yu. Kiselev, A. A. Cernant, Application of the B. N. Zhemochkin method for joint calculation of the "foundation-foundation-building" system, Online journal "SCIENCE STUDIES", tome 9, №4,128-137 (2017)

28. S. V. Bosakov, Application of B.N. Zhemochkin's Method to Analysis of a Bendable Slab on an Elastic Bed, Soil Mechanics and Foundation Engineering, Vol.40, issue 2, 48-54 (2003) [DOI: 10.1023/A:102448001627]

29. O. V. Kozunova, Application of MKR in nonlinear calculations of beams on a homogeneous elastic layer, Resource-saving materials, structures, buildings and structures, issue 17, 373-381 (2008) 